\title{
Experiences of healthcare providers with a novel emergency response intubation team during COVID-19
}

\author{
Daniel D. Lee ${ }^{1}(1) \cdot$ Matthew Hacker Teper ${ }^{1} \cdot$ Lucas B. Chartier $^{2,3} \cdot$ Stephanie Crump ${ }^{2} \cdot$ Martin Ma $^{4,5}$. \\ Matteo Parotto ${ }^{4,5,6} \cdot$ Pauline Perri $^{2} \cdot$ Ki Jinn Chin ${ }^{4,5} \cdot$ Konika Nirmalanathan $^{2} \cdot$ Sam Sabbah ${ }^{2,3} \cdot$ Ahmed K. Taher $^{2,3}$
}

Received: 5 July 2021 / Accepted: 6 December 2021 / Published online: 18 January 2022

(c) The Author(s), under exclusive licence to Canadian Association of Emergency Physicians (CAEP)/ Association Canadienne de Médecine d'Urgence (ACMU) 2022

\begin{abstract}
Objectives In the early stages of the COVID-19 pandemic, there were significant concerns about the infectious risks of intubation to healthcare providers. In response, a dedicated emergency response intubation team (ERIT) consisting of anesthesiologists and allied health providers was instituted for our emergency department (ED). Given the high-risk nature of intubations and the new interprofessional team dynamics, we sought to assess health-care provider experiences and potential areas of improvement.

Methods Surveys were distributed to healthcare providers at the University Health Network, a quaternary healthcare centre in Toronto, Canada, which includes two urban EDs seeing over 128,000 patients per year. Participants included ED physicians and nurses, anesthesiologists, anesthesia assistants, and operating room nurses. The survey included free-text questions. Responses underwent thematic analysis using grounded theory and were independently coded by two authors to generate descriptive themes. Discrepancies were resolved with a third author. Descriptive themes were distilled through an inductive, iterative process until fewer main themes emerged.

Results A total of 178 surveys were collected (68.2\% response rate). Of these, 123 (69\%) participated in one or more ERIT activations. Positive aspects included increased numbers of staff to assist, increased intubation expertise, improved safety, and good team dynamics within the ERIT team. Challenges included a loss of scope (primarily ED physicians and nurses) and unfamiliar workflows, perceived delays to ERIT team arrival or patient intubation, role confusion, handover concerns, and communication challenges between ED and ERIT teams. Perceived opportunities for improvement included interprofessional training, developing clear guidelines on activation, inter-team role clarification, and guidelines on handover processes post-intubation.

Conclusions Healthcare providers perceived that a novel interprofessional collaboration for intubations of COVID-19 patients presented both benefits and challenges. Opportunities for improvement centred around interprofessional training, shared decision making between teams, and structured handoff processes.
\end{abstract}

Keywords COVID-19 $\cdot$ Endotracheal intubation $\cdot$ Rapid response team

\section{Résumé}

Objectifs Aux premiers stades de la pandémie de COVID-19, les risques infectieux de l'intubation pour les prestataires de soins de santé ont suscité de vives inquiétudes. En réponse, une équipe d'intervention d'urgence en intubation (emergency response intubation team ERIT), composée d'anesthésistes et de prestataires de services paramédicaux, a été mise en place dans notre service d'urgence. Compte tenu de la nature à haut risque des intubations et de la nouvelle dynamique d'équipe

Presented at the Canadian Association of Emergency Physicians Conference, Winnipeg, MB, Canada, June 2021 (virtual).

Daniel D. Lee

Dongjoo.Lee@mail.utoronto.ca

Extended author information available on the last page of the article 
interprofessionnelle, nous avons cherché à évaluer les expériences des prestataires de soins et les domaines d'amélioration potentiels.

Méthodes Les questionnaires ont été distribués aux prestataires de soins de santé du University Health Network, un centre de soins de santé quaternaire de Toronto, au Canada, qui comprend deux urgences urbaines accueillant plus de 128000 patients par an. Les participants comprenaient des médecins et des infirmiers des urgences, des anesthésistes, des assistants en anesthésie et des infirmiers de salle d'opération. Les réponses ont fait l'objet d'une analyse thématique fondée sur la théorie de la base et ont été codées indépendamment par deux auteurs afin de générer des thèmes descriptifs. Les divergences ont été résolues avec un troisième auteur. Les thèmes descriptifs ont été distillés par un processus inductif et itératif jusqu'à ce qu'un nombre réduit de thèmes principaux émerge.

Résultats Au total, 178 sondages ont été recueillis (taux de réponse de 68,2\%). Parmi ceux-ci, 123 (69\%) ont participé à une ou plusieurs activations d'ERIT. Les aspects positifs comprenaient un nombre accru de personnel pour aider, une expertise accrue en matière d'intubation, une sécurité améliorée et une bonne dynamique d'équipe au sein de l'équipe ERIT. Parmi les difficultés rencontrées, citons la perte du champ d'action (principalement les médecins et les infirmières des services d'urgence) et les flux de travail non familiers, les retards perçus dans l'arrivée de l'équipe d'ERIT ou l'intubation du patient, la confusion des rôles, les problèmes de transfert et les difficultés de communication entre les équipes des services d'urgence et d'ERIT. Les possibilités d'amélioration perçues comprennent la formation interprofessionnelle, l'élaboration de directives claires sur l'activation, la clarification des rôles entre les équipes et les directives sur les processus de transfert après l'intubation.

Conclusions Les prestataires de soins de santé ont perçu qu'une nouvelle collaboration interprofessionnelle pour les intubations des patients COVID-19 présentait à la fois des avantages et des défis.

\section{Clinician's capsule}

\section{What is known about the topic?}

Many institutions formed dedicated emergency response intubation teams during COVID-19 that performed endotracheal intubations hospital-wide, including in the emergency department (ED).

\section{What did this study ask?}

What were the experiences of healthcare providers, including ED personnel, with a novel emergency response intubation team?

\section{What did this study find?}

Participants identified inconsistencies with training and challenges with communication and handover, shared decision making, and perceived delays in care.

\section{Why does this study matter to clinicians?}

Training, communication and handover, and shared decision-making are high-yield areas to improve interprofessional emergency airway management teams.

\section{Background}

During the early part of the COVID-19 pandemic, emergency departments (EDs) implemented changes to protect against the unknown safety risks of aerosol-generating medical procedures, such as endotracheal intubations [1]. These included specified "closed" negative pressure rooms, updated intubation protocols, and new aerosol containment devices [2]. In some hospitals, dedicated emergency response intubation teams were created to perform all inhospital intubations, including the ED where previously, intubations were performed by ED staff. These teams included anesthesiologists, anesthesia assistants, respiratory therapists, and other healthcare workers [3-5].

While hospital-wide airway teams have been described before, often in the context of difficult airways, COVID-19 presents unique considerations around infection control as well as provider and patient safety [6, 7]. As a novel collaboration in acute care, there is also potential for new communication and execution errors that may harm patients [8-11]. Moreover, timely activation and transport of critical equipment across a wide geographic area add further potential challenges.

Given the importance of provider and patient safety in airway management of COVID-19 patients, it is important to delineate any challenges and opportunities to optimize the emergency response intubation process. The objective of this study is to describe the experiences of both emergency response intubation team members and ED staff at a large urban academic hospital. Insights in emergency response intubation process improvement may be applicable for the COVID-19 pandemic as well as similar programs during future infectious pandemics.

\section{Methods}

\section{Study design}

We conducted a qualitative descriptive study with thematic analysis of free-text survey responses [12, 13]. Sampling, survey development and administration were guided 
by methods described by Burns et al. [14]. This study was approved by the University Health Network (UHN) Research Ethics Board (\#20-5638).

\section{Study setting, population and recruitment}

Study participants included ED nurses, ED physicians, anesthesiologists, anesthesia assistants and operating room nurses who regularly participated in emergency response intubation activations in the ED at UHN hospital sites. UHN is a quaternary healthcare centre in Toronto, Canada, which includes two urban EDs that together see up to 128,000 patients per year. Eligible participants were invited to participate in a qualitative survey administered either online or inperson. A stratified maximum variation sampling approach was used to ensure that perspectives from all members of the ED and emergency response intubation team were included [15].

\section{Emergency response intubation protocol}

At the onset of COVID-19, our institution developed a dedicated 24/7 emergency response intubation protocol for airway management of COVID-19 suspected or confirmed patients, including in ICUs and EDs. Each team comprised a staff anesthesiologist, two operating room registered nurses, one anesthesia assistant, and one personal care attendant. Dedicated equipment was pre-packed and brought by the emergency response intubation teams to the airway management location. Team members underwent simulation training prior to the establishment of the team. Teams would meet at the beginning of each shift to review treatment algorithms and simulate clinical scenarios, including donning and doffing of personal protective equipment.

\section{Data collection}

Participant perspectives were collected via a structured, qualitative, free-text survey. This survey was co-constructed by members of the research team and pilot tested with at least one participant from every group included in the study [14]. Participants were asked to describe (1) the training they received with the new emergency response intubation protocol; (2) their satisfaction with emergency response intubation team lead intubations; (3) their perceptions of any benefits and/or drawbacks of the new emergency response intubation protocol; and (4) their ideas for what could be improved in subsequent iterations of the emergency response intubation protocol. To minimize the risk that survey responses were biased or coerced, in-person surveys were administered by peer "clinical champions," who had no professional or authoritative power over participants.

\section{Data analysis}

Quantitative data on professional background and emergency response intubation team participation were tabulated. Survey responses were transcribed electronically and uploaded onto NVivo software (Version 12, QSR International Pty Ltd., Doncaster, Australia). Analysis was inductive to ensure that derived themes emerged directly from the data [16]. Line-by-line coding was completed independently by two members of the research team with experience in qualitative analysis (DL, AT). After coding, these researchers conducted a semantic thematic analysis, as defined by Braun and Clarke, to identify recurrent concepts, themes and ideas throughout the entire dataset [13]. Content validation then occurred, as primitive themes were discussed, debated and refined by the remaining members of the research team (LBC, SC, KN, MHT).

\section{Strategies to ensure trustworthiness}

Validity and trustworthiness were ensured by several factors. First, data collection tool development and content validation were completed by research team members with a wide range of professional backgrounds and experiences, including three emergency physicians, two emergency nurses, three anesthesiologists and two medical students. Second, we conducted member checking with two participants from every professional subgroup to allow participants to validate identified themes and to further elaborate on nuances or subthemes that our initial analysis did not identify $[17,18]$. Finally, we ensured a thorough audit trail of research [19].

\section{Results}

A total of 176 (68\%) of a potential 261 participants completed a qualitative survey. This included responses from 93 ED nurses (72\%), 49 ED physicians (70\%), 16 anesthesiologists (62\%), 6 anesthesia assistants (35\%) and 14 operating room RNs (78\%) (Table 1). Participants were approximately evenly distributed across the two EDs of UHN. The number of emergency response intubation participants involved is outlined in Table 1.

Participants spoke regularly about differences in training with the new emergency response intubation protocols, variability in team arrival time, the impact of the new emergency response intubation protocol changing their roles in the intubation process, improved safety with the new emergency response intubation protocol, and challenges with interprofessional communication. In each of these areas, participants suggested potential avenues for subsequent improvement of the emergency response intubation protocol. 
Table 1 Professional backgrounds and participation rates of participants

\begin{tabular}{|c|c|c|c|}
\hline Professional role & $\begin{array}{l}\text { How many ERITs have } \\
\text { you participated in? }\end{array}$ & Number & $(\%)$ \\
\hline \multirow[t]{6}{*}{ ED nurses } & 0 & 21 & 23 \\
\hline & 1 & 11 & 13 \\
\hline & $2-3$ & 33 & 41 \\
\hline & $4-5$ & 19 & 28 \\
\hline & $6-10$ & 8 & 14 \\
\hline & $11+$ & 1 & 2 \\
\hline \multirow[t]{6}{*}{ ED physicians } & 0 & 14 & 29 \\
\hline & 1 & 5 & 10 \\
\hline & $2-3$ & 21 & 43 \\
\hline & $4-5$ & 7 & 14 \\
\hline & $6-10$ & 2 & 4 \\
\hline & $11+$ & 0 & 0 \\
\hline \multirow[t]{6}{*}{ Anesthesia assistants } & 0 & 0 & 0 \\
\hline & 1 & 0 & 0 \\
\hline & $2-3$ & 1 & 20 \\
\hline & $4-5$ & 1 & 20 \\
\hline & $6-10$ & 3 & 60 \\
\hline & $11+$ & 0 & 0 \\
\hline \multirow[t]{6}{*}{ OR nurses } & 0 & 1 & 7 \\
\hline & 1 & 0 & 0 \\
\hline & $2-3$ & 2 & 14 \\
\hline & $4-5$ & 5 & 36 \\
\hline & $6-10$ & 6 & 43 \\
\hline & $11+$ & 0 & 0 \\
\hline \multirow[t]{6}{*}{ Anesthesiologists } & 0 & 2 & 13 \\
\hline & 1 & 0 & 0 \\
\hline & $2-3$ & 2 & 13 \\
\hline & $4-5$ & 6 & 38 \\
\hline & $6-10$ & 4 & 25 \\
\hline & $11+$ & 2 & 13 \\
\hline
\end{tabular}

$E D$ emergency department; ERIT emergency response intubation team; OR operating room

\section{Theme 1: discrepancy in training}

While emergency response intubation team protocols were rapidly and universally implemented across UHN EDs, participants reported that their training in these new protocols was highly variable. Participants endorsed a range of training experiences, including self-studying, receiving didactic teaching sessions from superiors, holding "conceptual discussions" with emergency response intubation teams, watching simulations, and participating in simulations.

Training, however, was not equally distributed across participant groups. While almost every anesthesiologist, anesthesia assistant and operating room RNs received formal simulation-based training, most ED physicians and nurses lacked formal training regarding emergency response intubation activations. As a result, ED staff reported feeling ill-prepared for real-world activations (Table 2, Subtheme 1A). By contrast, those participants who did participate in simulation training reported feeling better prepared (Table 2, Subtheme 1B). Specific training on donning and doffing was viewed as particularly valuable by non-ED staff participants.

\section{Theme 2: duration of time from activation to team arrival}

After a decision was made to intubate a patient and the emergency response intubation team was activated, some ED personnel perceived an increased length of time between team activation and the physical intubation process. As a result of these perceived delays, ED personnel reported experiencing a tension between following hospital protocols and concern for possible patient deterioration (Table 2, Theme 2).

\section{Theme 3: benefits of a cohesive and prepared emergency response intubation team}

Participants from all groups reported being impressed with operational speed, smoothness, and efficiency that emergency response intubation teams demonstrated during intubations. ED staff especially appreciated how the teams arrived with their own medication, personal protective equipment and tools. Stepping back from intubations allowed ED nurses and physicians to feel safer and to address other patient needs across a busy ED. (Table 2, Subtheme 3A). Anesthesiologists, anesthesia assistants and operating room RNs reported that having increased familiarity with one another on the emergency response intubation team allowed for greater efficiency and patient safety (Table 2, Subtheme 3B).

\section{Theme 4: ED staff's challenges in transitioning to a passive role}

While emergency physicians and nurses expressed gratitude for the emergency response intubation team's assistance in intubation, they also reported hesitancy in adopting more "passive" roles with the new protocol. Once the team arrived, many of the ED staff left the room for the intubation. Others transitioned to more of a "supportive" role or stayed to "keep an eye on the patient". Relinquishing active care of the patient was found to be difficult for many ED physicians, both in terms of their engagement with the intubation and adjusting to their new roles (Table 2, Subtheme 4A). ED nurses generally assisted the emergency response intubation team as runners or by documenting the encounter. They too struggled to establish coherence between their previous and new roles (Table 2, Subtheme 4B). 


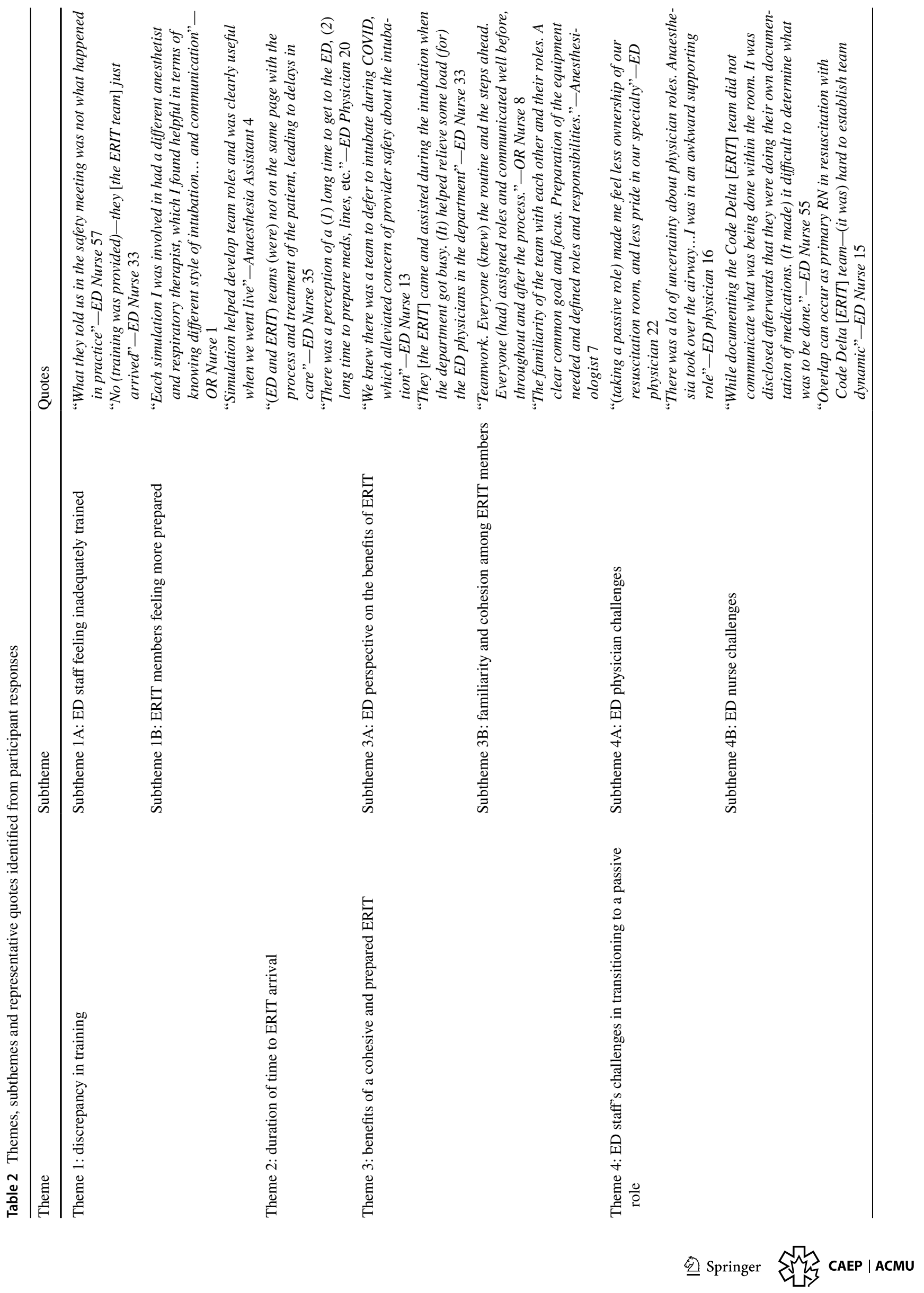




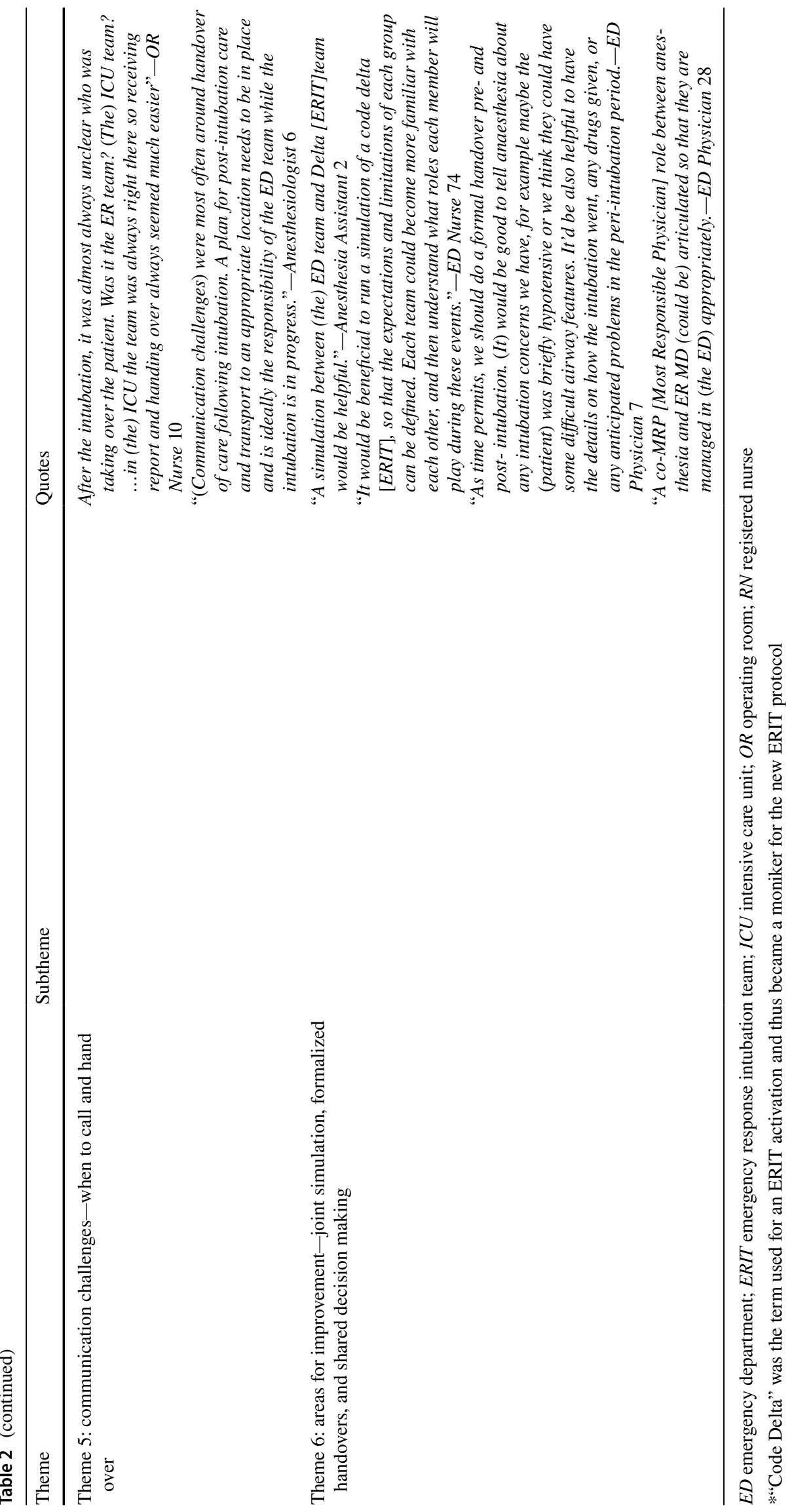




\section{Theme 5: communication challenges-when to call and handover}

Communication challenges occurred at several points throughout the emergency response intubation team activation process. For example, multiple participants reported that criteria for activation were misunderstood or miscommunicated. Others reported differences in communication styles between individuals and teams. Finally, some felt like communication difficulties were exacerbated when there were too many people with undefined roles participating in the intubation. These challenges notwithstanding, the most universally reported communication challenge by participants was related to post-intubation handover and ICU admission (Theme 5).

\section{Theme 6: areas for improvement-joint simulation, formalized handovers, and shared decision making}

When invited to suggest improvements for emergency response intubation team protocols in their departments, participants expressed optimism that small modifications rollout could improve the fluidity of the intubation process. Specifically, participants recommended three improvements (Theme 6). First, participants in both ED and emergency response intubation teams recommended instituting joint simulation training inclusive of both teams, to ensure that every stakeholder in the intubation process understands the roles of those around them. Second, participants articulated the need for a formalized handover procedure both pre- and post-intubation. This involves clarifying who is the most responsible physician for the patient at various time points. Finally, some ED physicians advocated for more of a "shared decision model", where the ED physicians play a more active role in determining the urgency of an intubation and work with anesthesiologists to establish an optimal care plan based on patient vital status and comorbidities.

\section{Discussion}

\section{Interpretation of findings}

Overall, this study describes the perceptions of ED and emergency response intubation team members with a new protected intubation protocol introduced in our hospital. A large proportion of potential participants from all involved professions were included. Of note, individual ED staff reported being involved in fewer team activations compared to the team members themselves. This inexperience may have contributed to the ED staff's challenges expressed in the qualitative data.
Participants emphasized the benefits of the new protocol on patient and provider safety by having a well-organized team with clearly defined roles and familiarity with one another. However, participants also articulated several challenges in adapting to the new protocol. Specifically, ED personnel felt underprepared for emergency response intubation activations, citing insufficient training on the new protocol. They also struggled to adapt to a more supportive role in patient care. Emergency response intubation team members most notably reported struggling with patient handovers and understanding who was responsible for patients after the intubation.

Participants' recommendations reflected two basic needs of both ED and emergency response intubation team members: increased interprofessional familiarity leading to improved communication; and, a more formalized understanding of the roles and responsibilities of each team member. Specifically, participants suggested simulation-based educational methods to learn team roles and workflow.

Perceived delays in intubation were only reported by ED staff, and no quantitative data on times were captured. Prior work has shown that time perception may be inaccurate during resuscitation events [20]. Moreover, each team may not have been aware of the other teams' workflow and practice patterns, exacerbating differences in expectations. Increasing exposure and familiarity with the other teams' processes may close this perception gap. Process mapping of the entire protocol may also identify activation errors or potential delays if present. Shortly after this survey was conducted, the emergency response intubation team leadership team began implementing in-situ simulation to build familiarity and competence in real working environments [21, 22].

Another concern that arose was the maintenance of airway skills for emergency physicians. The uncertain risk of aerosol-generating medical procedures to healthcare providers early in the pandemic, as well as a rapidly evolving intubation protocol, favored having one hospital-wide intubation team. It is nevertheless important for long-term patient safety that emergency physicians be skilled in airway management. This is especially true given that intubation success rates are correlated with provider experience [23].

\section{Strengths and limitations}

This study represents a first opportunity to understand the perceived challenges and areas of improvement regarding a novel emergency response intubation team protocol. This study is strengthened by a robust sample size across participant groups, and a systematic approach to collect and analyze responses.

Limitations exist as well. This study was done at a single quaternary care centre and the results may not be reliable to other hospital settings. Although there were similar response 
rates across most groups, there was a difference in absolute numbers of responses from the ED team and the emergency response intubation team. To minimize bias toward a higher numbered group, themes were derived from data from each separate group of providers before comparing across groups. Still, we note that anesthesia assistants are relatively underrepresented in our study population for unclear reasons. Furthermore, ICU providers were not included due to the study aim of understanding the experiences of healthcare providers in the ED. Themes emerged regarding handover concerns, which highlight the need for subsequent evaluations involving ICU providers to better address post-intubation care and handovers.

Finally, due to the free-text nature of data collection, participants may have not fully expanded on aspects of certain experiences as would be the case in other qualitative methods. While this had the advantage of ensuring a wide range of responses, each individual response is likely less reliable. This was mitigated by crafting and pilot testing our questionnaire with an interdisciplinary group of healthcare professionals, and by conducting member checking to ensure accurate interpretation of participant responses.

\section{Clinical implications}

Recent research has demonstrated that aerosol-generating medical procedures such as intubations incur less risk to healthcare providers than previously believed [32]. However, multidisciplinary care pathways in the ED are complex and face well-established safety challenges in communication and handover [24-26]. Accordingly, addressing perceived gaps in knowledge, dissemination, team roles, and inter-professional communication is essential [27, 28]. In addition to simulation training mentioned previously, other solutions for improved communication include training programs on cultural humility, conflict resolution, reinforcing aligned objectives that attend to skills specific to each discipline, and post-intubation clinical or "hot" debriefs [26, 27, 29-31]. The lessons learned from this rapidly implemented and high-acuity collaboration will remain pertinent as external personnel continue to play active roles in ED care [32].

\section{Research implications}

Our study represents a novel attempt to describe the experiences of healthcare professionals tasked with adjusting to a rapidly disseminated protocol during the COVID-19 pandemic. These results contribute to a small but growing body of literature comprising previous studies describing the implementation, operation, safety, and efficacy of emergency response intubation teams [3-5, 33-35]. Our findings highlight the need for evaluating the subjective experience of healthcare providers when transitioning to new models of care in response to emergencies. These considerations are crucial for implementing initiatives involving multidisciplinary teams.

\section{Conclusion}

The COVID-19 pandemic has challenged our healthcare system in unprecedented ways. This study represents a first attempt to describe the perspectives of healthcare professionals experiencing the introduction of emergency response intubation teams. Given the ongoing nature of COVID-19 and possible future transmissible pandemics, these findings will help inform the creation of future interdisciplinary protocols that involve airway management.

\section{Acknowledgements None.}

Author contributions DL: study concept and design, interpretation of the data, drafting of the manuscript, critical revision of the manuscript for important intellectual content. MHT: interpretation of the data, drafting of the manuscript, critical revision of the manuscript for important intellectual content. LBC: study concept and design, acquisition of the data, critical revision of the manuscript for important intellectual content. SC: acquisition of the data, critical revision of the manuscript for important intellectual content. MM: study concept and design, critical revision of the manuscript for important intellectual content. MP: study concept and design, critical revision of the manuscript for important intellectual content. PP: acquisition of the data, critical revision of the manuscript for important intellectual content. KJC: study concept and design, critical revision of the manuscript for important intellectual content. KN: study concept and design, acquisition of the data. SS: critical revision of the manuscript for important intellectual content. AKT: study concept and design, data acquisition, analysis and interpretation of the data, drafting of the manuscript, critical revision of the manuscript for important intellectual content.

\section{Declarations}

Conflict of interest None of the authors have potential conflicts of interest or have received financial support for this manuscript. All authors meaningfully contributed to revisions, have reviewed the final manuscript and have provided permission to publish the manuscript.

\section{References}

1. Weissman DN, de Perio MA, Radonovich LJ. COVID-19 and risks posed to personnel during endotracheal intubation. JAMA J Am Med Assoc. 2020;323(20):2027-8.

2. Simpson JP, Wong DN, Verco L, Carter R, Dzidowski M, Chan PY. Measurement of airborne particle exposure during simulated tracheal intubation using various proposed aerosol containment devices during the COVID-19 pandemic. Anaesthesia. 2020;75(12):1587-95.

3. Ahmad I, Jeyarajah J, Nair G, Ragbourne SC, Vowles B, Wong DJ, El-Boghdadly K. A prospective, observational, cohort study of airway management of patients with COVID-19 by specialist tracheal intubation teams. Can J Anesth/J Can Anesth. 2021;68(2):196-203. 
4. Sajayan A, Arora N, Williamson A, Nair A. COVID intubation team (CIT)-an experience at a tertiary UK centre. Trends Anaesth Crit Care. 2020;33:27.

5. Miller L, Luković E, Wagener G. Guiding airway management and personal protective equipment for COVID-19 intubation teams. Br J Anaesth. 2020;125(3):e288-90.

6. Mark LJ, Herzer KR, Cover R, Pandian V, Bhatti NI, Berkow LC, Haut ER, Hillel AT, Miller CR, Feller-Kopman DJ, Schiavi AJ. Difficult airway response team: a novel quality improvement program for managing hospital-wide airway emergencies. Anesth Analg. 2015;121(1):127.

7. Long L, Vanderhoff B, Smyke N, Shaffer LE, Solomon J, Steuer JD. Management of difficult airways using a hospital-wide "Alpha Team” approach. Am J Med Qual. 2010;25(4):297-304.

8. Gokhman R, Seybert AL, Phrampus P, Darby J, Kane-Gill SL. Medication errors during medical emergencies in a large, tertiary care, academic medical center. Resuscitation. 2012;83(4):482-7.

9. Joint Commission T. Improving America's Hospitals The Joint Commission's Annual Report on Quality and Safety 2007 [Internet]. 2007 [Cited 14 Jun 2021]. Available from: www.jointcommi ssionreport.org

10. Wilson RM, Runciman WB, Gibberd RW, Harrison BT, Newby L, Hamilton JD. The quality in Australian health care study. Med J Aust. 1995;163(9):458-71.

11. Solet DJ, Norvell JM, Rutan GH, Frankel RM. Lost in translation: challenges and opportunities in physician-to-physician communication during patient handoffs. Acad Med. 2005;80(12):1094-9.

12. Sandelowski M. Whatever happened to qualitative description? Res Nurs Health. 2000;23(4):334-40.

13. Braun V, Clarke V. Using thematic analysis in psychology. Qual Res Psychol. 2006;3(2):77-101.

14. Burns KE, Duffett M, Kho ME, Meade MO, Adhikari NK, Sinuff T, Cook DJ. A guide for the design and conduct of self-administered surveys of clinicians. CMAJ. 2008;179(3):245-52.

15. Marshall MN. Sampling for qualitative research. Fam Pract. 1996;13(6):522-6.

16. Saldaña J. The Coding Manual for Qualitative Researchers. 3rd ed. London (GB): Sage; 2016.

17. Morse JM. Critical analysis of strategies for determining rigor in qualitative inquiry. In: Qualitative health research. SAGE Publications Inc.; 2015. p. 1212-22.

18. Groleau D, Zelkowitz P, Cabral EI. Enhancing generalizability: moving from an intimate to a political voice. Qual Health Res. 2009;19(3):416-26.

19 Shenton AK. Strategies for ensuring trustworthiness in qualitative research projects. IOS Press; 2004.

20. Trevisanuto D, De Bernardo G, Sordino D, Doglioni N, Weiner G, Cavallin F. Time perception during neonatal resuscitation. J Pediatr. 2016;177:103-7.
21. Petrosoniak A, Auerbach M, Wong AH, Hicks CM. In situ simulation in emergency medicine: moving beyond the simulation lab. EMA Emerg Med Australas. 2017;29(1):83-8.

22. Patterson MD, Geis GL, Falcone RA, LeMaster T, Wears RL. In situ simulation: detection of safety threats and teamwork training in a high risk emergency department. BMJ Qual Saf. 2013;22(6):468-77.

23. Garza AG, Gratton MC, Coontz D, Noble E, Ma OJ. Effect of paramedic experience on orotracheal intubation success rates. $\mathrm{J}$ Emerg Med. 2003;25(3):251-6.

24. Shaw KN, Lillis KA, Ruddy RM, et al. Reported medication events in a paediatric emergency research network: sharing to improve patient safety. Emerg Med J. 2013;30(10):815-9.

25. Jepson ZK, Darling CE, Kotkowski KA, Bird SB, Arce MW, Volturo GA, Reznek MA. Emergency department patient safety incident characterization: an observational analysis of the findings of a standardized peer review process. BMC Emerg Med. 2014;14(1):1-7.

26. Fordyce J, Blank FS, Pekow P, Smithline HA, Ritter G, Gehlbach S, Benjamin E, Henneman PL. Errors in a busy emergency department. Ann Emerg Med. 2003;42(3):324-33.

27. Foronda C, MacWilliams B, McArthur E. Interprofessional communication in healthcare: an integrative review. Nurse Educ Pract. 2016;19:36-40.

28. Wagner J, Liston B, Miller J. Developing interprofessional communication skills. Teach Learn Nurs. 2011;6(3):97-101.

29. Friend ML, Friend RD, Ford C, Ewell PJ. Critical care interprofessional education: exploring conflict and power-lessons learned. J Nurs Educ. 2016;55(12):696-700.

30. Rose $\mathrm{S}$, Cheng A. Charge nurse facilitated clinical debriefing in the emergency department. Can J Emerg Med. 2018;20(5):781-5.

31. Fanning RM, Gaba DM. The role of debriefing in simulationbased learning. Simul Healthcare. 2007;2(2):115-25.

32. Klompas M, Baker M, Rhee $\mathrm{C}$. What is an aerosol-generating procedure? JAMA Surg. 2021;156(2):113-4.

33. Shrestha A, Shrestha A, Sonnenberg T, Shrestha R. Covid-19 emergency department protocols: experience of protocol implementation through in-situ simulation. Open Access Emerg Med. 2020;12:293-303.

34. Yao W, Wang T, Jiang B, et al. Emergency tracheal intubation in 202 patients with COVID-19 in Wuhan, China: lessons learnt and international expert recommendations. Br J Anaesth. 2020;125(1):e28-37.

35. Bai JW, Ravi A, Notario L, Choi M. Opening the discussion on a closed intubation box. Trends Anaesth Crit Care. 2020;33:1-4.

\section{Authors and Affiliations}

\section{Daniel D. Lee ${ }^{1} \oplus$ - Matthew Hacker Teper ${ }^{1}$ - Lucas B. Chartier ${ }^{2,3}$. Stephanie Crump ${ }^{2}$ - Martin Ma ${ }^{4,5}$. Matteo Parotto ${ }^{4,5,6} \cdot$ Pauline Perri $^{2} \cdot$ Ki Jinn Chin $^{4,5} \cdot$ Konika Nirmalanathan $^{2} \cdot$ Sam Sabbah $^{2,3} \cdot$ Ahmed K. Taher $^{2,3}$}

Matthew Hacker Teper

Matthew.Hackerteper@mail.utoronto.ca

Lucas B. Chartier

Lucas.Chartier@uhn.ca

Stephanie Crump

Stephanie.Crump@uhn.ca

Martin Ma

Martin.Ma@uhn.ca
Matteo Parotto

Matteo.Parotto@uhn.ca

Pauline Perri

Pauline.Perri@uhn.ca

Ki Jinn Chin

KiJinn.Chin@uhn.ca

Konika Nirmalanathan

Konika.Nirmalanathan@uhn.ca 
Sam Sabbah

Sam.Sabbah@uhn.ca

Ahmed K. Taher

Ahmed.Taher@mail.utoronto.ca

1 Temerty Faculty of Medicine, University of Toronto, Toronto, ON, Canada

2 Department of Emergency Medicine, University Health Network, Toronto, ON, Canada
3 Division of Emergency Medicine, Department of Medicine, University of Toronto, Toronto, ON, Canada

4 Department of Anesthesia and Pain Medicine, University of Toronto, Toronto, ON, Canada

5 Department of Anesthesia and Pain Management, University Health Network, Toronto, ON, Canada

6 Interdepartmental Division of Critical Care Medicine, University of Toronto, Toronto, ON, Canada 